\title{
ON AN INVARIANT SUBMANIFOLD OF HYPERBOLIC SASAKIAN MANIFOLDS
}

\author{
Shravan Kumar Pandey and Ram Nawal Singh
}

\begin{abstract}
The objective of the present paper is to study an invariant submanifold of hyperbolic Sasakian manifolds. In this paper, we consider semiparallel and 2-semiparallel invariant submanifolds of hyperbolic Sasakian manifolds and shown that these submanifolds are totally geodesic. It is also proved that on an invariant submanifold of hyperbolic Sasakian manifolds the condition $I(X, Y) \cdot \alpha=0, I(X, Y) \cdot \tilde{\nabla} \alpha=0, C(X, Y) \cdot \alpha=0$ and $C(X, Y) . \tilde{\nabla} \alpha=0$ holds if and only if it is totally geodesic.

Keywords: hyperbolic Sasakian manifold, invariant submanifold, semiparallel submanifold, 2-semiparallel submanifold, totally geodesic submanifold
\end{abstract}

\section{Introduction}

In 1969, Blair and Ludden [3] studied the hypersurfaces in an almost contact manifolds. Goldberg and Yano [11] studied the non-trivial hypersurfaces of almost contact manifolds. Sinha and Sharma [23] studied the hypersurfaces of almost paracontact metric manifold with para $(f, g, u, v, \lambda)$ structure.

Mishra studied the submanifold of a locally product Riemannian manifold and an almost complex manifold and almost contact submanifolds in 1968 [15] and 1972 [16], respectively. In 1971, Goldberg [12] studied the invariant submanifolds of codimension 2 of almost contact manifolds. The globally framed f-manifolds and their metric submanifolds have been studied by Mishra and Rathore [17].

Nowadays, the geometry of submanifolds have become a subject of growing interest for its significant application in applied mathematics and theoretical physics. For instance, the method of invariant submanifolds is used in the study of non-linear autonomous systems [13]. Also, the notion of geodesics plays an important role in the theory of relativity [14]. For totally geodesic submanifolds, the geodesics of ambient manifolds remain geodesics in the submanifolds. Hence, totally geodesic

Received November 20, 2014; accepted February 13, 2016

2010 Mathematics Subject Classification. Primary 53C25; Secondary 53C40 
submanifolds have also importance in physical sciences. The study of geometry of invariant submanifolds was initiated by A. Bejancu and N. Papaghuic [2]. Again, N. Papaghuic [19] has worked on semi-invariant submanifolds. On the other hand, a number of works on the geometry of submanifolds have been carried out by U.C. De and collaborators ([6],[7],[8],[9]). A. Sarkar [20], A.Sarakar and M.Sen [21], S.Sular and C. Özgur [24] and many others have worked on geometry of submanifolds.

The present paper is organized as follows. In Section 2, we recall the notion of hyperbolic Sasakian manifolds and the results related to submanifold theory. Section 3 is devoted to the study of semiparallel and 2-semiparallel invariant submanifolds of hyperbolic Sasakian manifolds. Section 4 contains the invariant submanifold of hyperbolic Sasakian manifold satisfying $I(X, Y) \cdot \alpha=0$ and $I(X, Y) \cdot \tilde{\nabla} \alpha=0$, where I is the concircular curvature tensor and $\alpha$ is the second fundamental form of the immersion. The last section admits the invariant submanifold of hyperbolic Sasakian manifold satisfying $C(X, Y) \cdot \alpha=0$ and $C(X, Y) \cdot \tilde{\nabla} \alpha=0$, where $\mathrm{C}$ is the conformal curvature tensor.

\section{Preliminaries}

Let $M$ be a complete real differentiable manifold of dimension $(2 \mathrm{n}+1)$. Let there exist a tensor field $\phi$ of type $(1,1)$, a vector field $\xi$ and a 1-form $\eta$ satisfying

$$
\begin{gathered}
\phi^{2} X=X+\eta(X) \xi, \\
\eta(\phi X)=0,
\end{gathered}
$$

for arbitrary vector fields $\mathrm{X}, \mathrm{Y} \in T M$. Then $\mathrm{M}$ is called a hyperbolic contact manifold $([23],[25])$. From the above equation we can easily prove that

$$
\begin{gathered}
\phi \xi=0, \\
\eta(\xi)=-1 .
\end{gathered}
$$

Let the hyperbolic contact manifold $\mathrm{M}$ be endowed with a pseudo-Riemannian metric $g$ such that

$$
\begin{gathered}
\Phi(X, Y)=g(\phi X, Y), \\
g(\phi X, \phi Y)=-g(X, Y)-\eta(X) \eta(Y), \\
g(X, \xi)=\eta(X) .
\end{gathered}
$$

A hyperbolic contact structure satisfying the equations (2.1) to (2.6) is said to be a hyperbolic contact metric manifold [25]. 
A hyperbolic contact metric manifold is said to be a hyperbolic cosymplectic metric manifold if the structure tensor $\phi$ and the 1-form $\eta$ are parallel with respect to a symmetric affine connection $\nabla$ on M. Since $\phi^{2}=I+\eta \otimes \xi$, the vector field $\xi$ is also parallel with respect to $\xi$, i.e.

$$
\begin{gathered}
\left(\nabla_{X} \phi\right)(Y)=0, \\
\left(\nabla_{X} \eta\right)(Y)=0, \\
\nabla_{X} \xi=0 .
\end{gathered}
$$

A hyperbolic contact metric manifold $\mathrm{M}$ in which

$$
-2 \Phi=d \eta
$$

is satisfied is called an almost hyperbolic Sasakian manifold.

An almost hyperbolic Sasakian manifold M, for which $\xi$ is Killing vector, i.e.

$$
\left(\nabla_{X} \eta\right)(Y)+\left(\nabla_{Y} \eta\right)(X)=0
$$

where $\nabla$ is the Riemannian connection, is called a hyperbolic K-contact Riemannian manifold.

In a hyperbolic K-contact Riemannian manifold, the following relations hold

$$
\begin{gathered}
\Phi(X, Y)=-\left(\nabla_{X} \eta\right)(Y)=\left(\nabla_{Y} \eta\right)(X), \\
\nabla_{X} \xi=-\phi X .
\end{gathered}
$$

A hyperbolic K-contact Riemannian manifold $\mathrm{M}$ is called a hyperbolic Sasakian manifold [23] if

$$
\left(\nabla_{X} \phi\right)(Y)=g(X, Y) \xi-\eta(Y) X \text {. }
$$

In a hyperbolic Sasakian manifold M the following relations hold

$$
\begin{gathered}
{ }^{\prime} R(X, Y, Z, \xi)=\eta(R(X, Y) Z)=g(Y, Z) \eta(X)-g(X, Z) \eta(Y), \\
\left(\nabla_{Z} \Phi\right)(X, Y)=g(X, Z) \eta(Y)-g(Y, Z) \eta(X), \\
\left(\nabla_{Z} \Phi\right)(X, Y)+\Phi(X, Y, Z, \xi)=0, \\
\left(\nabla_{X} \Phi\right)(Y, Z)+\left(\nabla_{Y} \Phi\right)(Z, X)+\left(\nabla_{Z} \Phi\right)(X, Y)=0 .
\end{gathered}
$$

Also, from equation (2.16), we have

$$
R(X, Y) \xi=\eta(Y) X-\eta(X) Y,
$$




$$
\begin{gathered}
R(\xi, Y) \xi=\eta(Y) \xi+Y, \\
S(Y, \xi)=(n-1) \eta(Y), \\
Q \xi=(n-1) \xi .
\end{gathered}
$$

Let $\tilde{M}$ be a submanifold immersed in a $(2 \mathrm{n}+1)$-dimensional Riemannian manifold M. Using the same symbol $g$, we denote the induced metric on $\tilde{M}$. Let $T \tilde{M}$ be a set of all vector fields tangent to $\tilde{M}$, and $T^{\perp} \tilde{M}$ is a set of all vector fields normal to $\tilde{M}$. Then the Gauss and Weingarten formulas are given by [5]

$$
\begin{aligned}
& \nabla_{X} Y=\tilde{\nabla}_{X} Y+\alpha(X, Y), \\
& \nabla_{X} N=-A_{N} X+\tilde{\nabla}_{X}^{\perp} N,
\end{aligned}
$$

for all vector fields $\mathrm{X}, \mathrm{Y} \in T \tilde{M}$ and $\mathrm{N} \in T^{\perp} \tilde{M}$, where $\tilde{\nabla}$ is the Riemannian connection on $\tilde{M}$ defined by the induced metric $g$, and $\tilde{\nabla}^{\perp}$ is the normal connection on $T^{\perp} \tilde{M}$ of $\tilde{M} ; \alpha$ is the second fundamental form of the immersion and $A_{N}$ is the shape operator with respect to a normal section $\mathrm{N}$. The second fundamental form $\alpha$ and $A_{N}$ are related by

$$
g(\alpha(X, Y), N)=g\left(A_{N} X, Y\right),
$$

for each $X, Y \in T \tilde{M}$ and $N \in T^{\perp} \tilde{M}$. It is also noted that $\alpha(X, Y)$ is bilinear in $\mathrm{X}$ and $\mathrm{Y}[5]$ and since $\tilde{\nabla}_{f X} Y=f \tilde{\nabla}_{X} Y$, for a $C^{\infty}$-function $\mathrm{f}$ on a manifold we have

$$
\alpha(f X, Y)=f \alpha(X, Y) .
$$

For the second fundamental form $\alpha$, the covariant derivative of $\alpha$ is defined by

$$
\left(\bar{\nabla}_{X} \alpha\right)(Y, Z)=\tilde{\nabla}_{X}^{\perp}(\alpha(Y, Z))-\alpha\left(\tilde{\nabla}_{X} Y, Z\right)-\alpha\left(Y, \tilde{\nabla}_{X} Z\right)
$$

for any vector fields $\mathrm{X}, \mathrm{Y}, \mathrm{Z}$ tangent to $\tilde{M}$. Then $\bar{\nabla} \alpha$ is a normal bundle valued tensor of type $(0,3)$ and is called the third fundamental form of $\tilde{M}$. $\bar{\nabla}$ is called the van der Waerden-Bortolotti connection of $\tilde{M}$, i.e. $\bar{\nabla}$ is a connection in $T \tilde{M} \oplus$ $T^{\perp} \tilde{M}$ built with $\tilde{\nabla}$ and $\tilde{\nabla}^{\perp}$. If $\bar{\nabla} \alpha=0$, then $\tilde{M}$ is said to have a parallel second fundamental form [5]. From the Gauss and Weingarten formulas, we obtain

$$
R(X, Y) Z=\tilde{R}(X, Y) Z+A_{\alpha(X, Z)} Y-A_{\alpha(Y, Z)} X .
$$

An immersion is said to be semiparallel [10], if

$$
\bar{R}(X, Y) \cdot \alpha=\left(\bar{\nabla}_{X} \bar{\nabla}_{Y}-\bar{\nabla}_{Y} \bar{\nabla}_{X}-\bar{\nabla}_{[X, Y]}\right) \cdot \alpha=0
$$

holds for all vector fields $\mathrm{X}, \mathrm{Y}$ tangent to $\tilde{M}$, where $\bar{R}$ denotes the curvature tensor of the connection $\bar{\nabla}$. 
In [1], the authors have defined and studied submanifolds satisfying the condition

$$
\bar{R}(X, Y) \cdot \bar{\nabla} \alpha=0
$$

for all vector fields $\mathrm{X}, \mathrm{Y} \in T \tilde{M}$. Submanifolds satisfying the equation (2.31) are called 2-semiparallel. Now, from the equation (2.30) we have

$$
(\bar{R}(X, Y) . \alpha)(U, V)=\tilde{R}^{\perp}(X, Y) \alpha(U, V)-\alpha(\tilde{R}(X, Y) U, V)-\alpha(U, \tilde{R}(X, Y) V),
$$

for all vector fields $\mathrm{X}, \mathrm{Y}, \mathrm{U}$ and $\mathrm{V}$ tangent to $\tilde{M}$, where

$$
\tilde{R}^{\perp}(X, Y)=\left[\tilde{\nabla}_{X}^{\perp}, \tilde{\nabla}_{Y}^{\perp}\right]-\tilde{\nabla}_{[X, Y]}^{\perp}
$$

and $\bar{R}$ denotes the curvature tensor of $\bar{\nabla}$.

Similarly, we have

$$
\begin{aligned}
(\bar{R}(X, Y) \cdot \bar{\nabla} \alpha)(U, V, Z) & =\tilde{R}^{\perp}(X, Y)(\bar{\nabla} \alpha)(U, V, Z)-(\bar{\nabla} \alpha)(\tilde{R}(X, Y) U, V, Z) \\
& -(\bar{\nabla} \alpha)(U, \tilde{R}(X, Y) V, Z)-(\bar{\nabla} \alpha)(U, V, \tilde{R}(X, Y) Z)
\end{aligned}
$$

for all vector fields tangent to $\tilde{M}$, where [1]

$$
(\bar{\nabla} \alpha)(U, V, Z)=\left(\bar{\nabla}_{U} \alpha\right)(V, Z) .
$$

Definition 2.1. Let $\tilde{M}$ be a submanifold of a hyperbolic Sasakian manifold M. The submanifold $\tilde{M}$ of $\mathrm{M}$ is said to be invariant if the structure vector field $\xi$ is tangent to $\tilde{M}$ at every point of $\tilde{M}$ and $\phi X$ is tangent to $\tilde{M}$ for any vector field $\mathrm{X}$ tangent to $\tilde{M}$ at every point of $\tilde{M}$, i.e. $\phi T \tilde{M} \subset T \tilde{M}$ at every point of $\tilde{M}$.

Definition 2.2. A submanifold $\tilde{M}$ of a hyperbolic Sasakian manifold $M$ is called totally geodesic if

$$
\alpha(X, Y)=0 \text { or equivalentally } A_{N}=0
$$

for all $\mathrm{X}, \mathrm{Y} \in T \tilde{M}$ and any $N \in T^{\perp} \tilde{M}$.

\section{Semiparallel and 2-Semiparallel Invariant Submanifolds of Hyperbolic Sasakian Manifolds}

Lemma 3.1. [22] For an invariant submanifold $\tilde{M}$ of a hyperbolic Sasakian manifold $M$, we have for the two differentiable tangent vectors $X, Y$ of $\tilde{M}$

$$
\begin{gathered}
\alpha(X, \xi)=0, \\
\alpha(X, \phi Y)=\phi \alpha(X, Y)=\alpha(\phi X, Y) .
\end{gathered}
$$


Lemma 3.2. [22] If $\tilde{M}$ is an invariant submanifold of a hyperbolic Sasakian manifold $M$. Then the following holds on $\tilde{M}$

$$
\begin{gathered}
\tilde{\nabla}_{X} \xi=-\phi X, \\
\tilde{R}(X, Y) \xi=\eta(Y) X-\eta(X) Y, \\
\tilde{R}(\xi, X) Y=g(X, Y) \xi-\eta(Y) X, \\
\tilde{Q} Y=(n-1) Y, \quad \tilde{Q} \xi=(n-1) \xi, \\
\tilde{S}(X, \xi)=(n-1) \eta(X),
\end{gathered}
$$

Proposition 3.1. [22] An invariant submanifold $\tilde{M}$ of a hyperbolic Sasakian manifold $M$ is also hyperbolic Sasakian.

Theorem 3.1. Let $\tilde{M}$ be an invariant submanifold of a hyperbolic Sasakian manifold $M$. Then $\tilde{M}$ is semiparallel if and only if it is totally geodesic.

Proof: Let $\tilde{M}$ be an invariant submanifold of a hyperbolic Sasakian manifold and let $\tilde{M}$ be semiparallel, i.e. $\bar{R} . \alpha=0$. Then from the equation (2.32), we have

$$
\tilde{R}^{\perp}(X, Y) \alpha(U, V)-\alpha(\tilde{R}(X, Y) U, V)-\alpha(U, \tilde{R}(X, Y) V)=0 .
$$

Taking $X=V=\xi$ in the above equation, we get

$$
\tilde{R}^{\perp}(\xi, Y) \alpha(U, \xi)-\alpha(\tilde{R}(\xi, Y) U, \xi)-\alpha(U, \tilde{R}(\xi, Y) \xi)=0 .
$$

In view of the equation (3.1), the above equation reduces to

$$
\alpha(U, \tilde{R}(\xi, Y) \xi)=0 .
$$

By virtue of the equation (3.5), the above equation takes the form

$$
\alpha(U, \xi) \eta(Y)+\alpha(U, Y)=0,
$$

which, on using the equation (3.1), gives

$$
\alpha(U, Y)=0 .
$$

This shows that $\tilde{M}$ is totally geodesic. The converse of the statement is trivial. This completes the proof.

Theorem 3.2. Let $\tilde{M}$ be an invariant submanifold of a hyperbolic Sasakian manifold $M$. Then $\tilde{M}$ has a parallel second fundamental form if and only if $\tilde{M}$ is totally geodesic. 
Proof: Since $\tilde{M}$ has a parallel second fundamental form, it follows from the equation (2.28), that

$$
\left(\bar{\nabla}_{X} \alpha\right)(Y, Z)=\tilde{\nabla}_{X}^{\perp}(\alpha(Y, Z))-\alpha\left(\tilde{\nabla}_{X} Y, Z\right)-\alpha\left(Y, \tilde{\nabla}_{X} Z\right)=0 .
$$

Now, putting $Z=\xi$ in the above equation and using the equation (3.1), we get

$$
-\alpha\left(Y, \tilde{\nabla}_{X} \xi\right)=0 .
$$

Thus in view of the equation (3.3), we have

$$
\alpha(Y, \phi X)=0 .
$$

Replacing $\mathrm{X}$ by $\phi X$ in the above equation and using the equations (2.1) and (3.1), we get

$$
\alpha(Y, X)=0,
$$

, which shows that $\tilde{M}$ is totally geodesic. The converse statement is trivial. This completes the proof.

Theorem 3.3. An invariant submanifold $\tilde{M}$ of a hyperbolic Sasakian manifold $M$ is 2-semiparallel if and only if $\tilde{M}$ is totally geodesic.

Proof: Let $\tilde{M}$ be a 2 -semiparallel, i.e. $\bar{R} \cdot \bar{\nabla} \alpha=0$. Then, in view of the equation (2.33), we have

$$
\begin{aligned}
& \tilde{R}^{\perp}(X, Y)(\bar{\nabla} \alpha)(U, V, Z)-(\bar{\nabla} \alpha)(\tilde{R}(X, Y) U, V, Z)-(\bar{\nabla} \alpha)(U, \tilde{R}(X, Y) V, Z) \\
& -(\bar{\nabla} \alpha)(U, V, \tilde{R}(X, Y) Z)=0 .
\end{aligned}
$$

Taking $X=V=\xi$ in the above equation, we get

$$
\begin{aligned}
& \tilde{R}^{\perp}(\xi, Y)(\bar{\nabla} \alpha)(U, \xi, Z)-(\bar{\nabla} \alpha)(\tilde{R}(\xi, Y) U, \xi, Z)-(\bar{\nabla} \alpha)(U, \tilde{R}(X, Y) V, Z) \\
& -(\bar{\nabla} \alpha)(U, \xi, \tilde{R}(\xi, Y) Z)=0 .
\end{aligned}
$$

By virtue of the equations (2.28), (2.32) and (3.1), we have the following equalities

$$
\begin{aligned}
(\bar{\nabla} \alpha)(U, \xi, Z) & =\left(\bar{\nabla}_{U} \alpha\right)(\xi, Z) \\
& =\tilde{\nabla}_{U}^{\perp}(\alpha(\xi, Z))-\alpha\left(\tilde{\nabla}_{U} \xi, Z\right)-\alpha\left(\xi, \tilde{\nabla}_{U} Z\right) \\
& =-\alpha\left(\tilde{\nabla}_{U} \xi, Z\right) .
\end{aligned}
$$

Now, using the equation (3.3) in the above equation, we get

$$
(\bar{\nabla} \alpha)(U, \xi, Z)=\alpha(\phi U, Z),
$$

$$
\begin{aligned}
(\bar{\nabla} \alpha)(\tilde{R}(\xi, Y) U, \xi, Z) & =\left(\bar{\nabla}_{\tilde{R}(\xi, Y) U} \alpha\right)(\xi, Z) \\
& =\tilde{\nabla}_{\tilde{R}(\xi, Y) U}^{\perp}(\alpha(\xi, Z))-\alpha\left(\tilde{\nabla}_{\tilde{R}(\xi, Y) U} \xi, Z\right)-\alpha\left(\xi, \tilde{\nabla}_{\tilde{R}(\xi, Y) U} Z\right) \\
& =-\alpha\left(\tilde{\nabla}_{\tilde{R}(\xi, Y) U} \xi, Z\right) \\
& =\alpha(\phi(\tilde{R}(\xi, Y) U), Z) .
\end{aligned}
$$


In view of the equation (3.5), the above equation takes the form

$$
(\bar{\nabla} \alpha)(\tilde{R}(\xi, Y) U, \xi, Z)=-\eta(U) \alpha(\phi Y, Z),
$$

$$
\begin{aligned}
(\bar{\nabla} \alpha)(U, \tilde{R}(\xi, Y) \xi, Z) & =\left(\bar{\nabla}_{U} \alpha\right)(\tilde{R}(\xi, Y) \xi, Z) \\
& =\tilde{\nabla}_{U}^{\perp}(\alpha(\tilde{R}(\xi, Y) \xi, Z))-\alpha\left(\tilde{\nabla}_{U} \tilde{R}(\xi, Y) \xi, Z\right) \\
& -\alpha\left(\tilde{R}(\xi, Y) \xi, \tilde{\nabla}_{U} Z\right),
\end{aligned}
$$

which, on using the equation (3.4), gives

$$
(\bar{\nabla} \alpha)(U, \tilde{R}(\xi, Y) \xi, Z)=\tilde{\nabla}_{U}^{\perp} \alpha(Y, Z)-\alpha\left(\tilde{\nabla}_{U}(\eta(Y) \xi+Y), Z\right)-\alpha\left(Y, \tilde{\nabla}_{U} Z\right) .
$$

Now

$$
\begin{aligned}
(\bar{\nabla} \alpha)(U, \xi, \tilde{R}(\xi, Y) Z)= & \left(\bar{\nabla}_{U} \alpha\right)(\xi, \tilde{R}(\xi, Y) Z) \\
= & \tilde{\nabla}_{U}^{\perp}(\alpha(\xi, \tilde{R}(\xi, Y) Z))-\alpha\left(\tilde{\nabla}_{U} \xi, \tilde{R}(\xi, Y) Z\right) \\
& -\alpha\left(\xi, \tilde{\nabla}_{U} \tilde{R}(\xi, Y) Z\right) \\
= & -\alpha\left(\tilde{\nabla}_{U} \xi, \tilde{R}(\xi, Y) Z\right) \\
= & \alpha(\phi U, \tilde{R}(\xi, Y) Z) .
\end{aligned}
$$

Using the equations (3.16), (3.18), (3.19) and (3.20) in equation (3.14), we get

$$
\begin{aligned}
& \tilde{R}^{\perp}(\xi, Y) \alpha(\phi U, Z)+\eta(U) \alpha(\phi Y, Z)+\tilde{\nabla}_{U}^{\perp} \alpha(Y, Z) \\
& +\alpha\left(\tilde{\nabla}_{U}(\eta(Y) \xi+Y), Z\right)+\alpha\left(Y, \tilde{\nabla}_{U} Z\right)-\alpha(\phi U, \tilde{R}(\xi, Y) Z)=0 .
\end{aligned}
$$

Taking $Z=\xi$ in the above equation and using the equation (3.1), we get

$$
\alpha(Y, \phi U)=0 .
$$

Putting $U=\phi U$ in the above equation and using the equations (2.1) and (3.1), we get

$$
\alpha(Y, U)=0,
$$

which shows that $\tilde{M}$ is totally geodesic. The converse part is obvious. This completes the proof.

\section{Invariant Submanifold of Hyperbolic Sasakian Manifolds Satisfying $\bar{I}(X, Y) \cdot \alpha=0$ and $\bar{I}(X, Y) \cdot \bar{\nabla} \alpha=0$.}

The concircular curvature tensor I of an n-dimensional Riemannian manifold is given by $[26]$

$$
I(X, Y) Z=R(X, Y) Z-\frac{r}{n(n-1)}[g(Y, Z) X-g(X, Z) Y],
$$


for all vector fields $\mathrm{X}, \mathrm{Y}$ and $\mathrm{Z}$ on $\mathrm{M}$, where $\mathrm{r}$ is the scalar curvature of $M$.

Putting $X=\xi$ in the above equation and using the equations (2.7) and (3.5), we get

$$
\tilde{I}(\xi, Y) Z=\left[1-\frac{\tilde{r}}{n(n-1)}\right][g(Y, Z) \xi-\eta(Z) Y],
$$

which gives

$$
\tilde{I}(\xi, Y) \xi=\left[1-\frac{\tilde{r}}{n(n-1)}\right][\eta(Y) \xi+Y]
$$

where $\tilde{I}$ is the concircular curvature tensor of $\tilde{M}$.

Similar to the equations (2.32) and (2.33) the tensors $\bar{I}(X, Y) . \alpha$ and $\bar{I}(X, Y) \cdot \bar{\nabla} \alpha$ are defined by $[18]$

and

$$
(\bar{I}(X, Y) . \alpha)(U, V)=\tilde{R}^{\perp}(X, Y) \alpha(U, V)-\alpha(\tilde{I}(X, Y) U, V)-\alpha(U, \tilde{I}(X, Y) V)
$$

$$
\begin{aligned}
(\bar{I}(X, Y) \cdot \bar{\nabla} \alpha)(U, V, Z) & =\tilde{R}^{\perp}(X, Y)(\bar{\nabla} \alpha)(U, V, Z)-(\bar{\nabla} \alpha)(\tilde{I}(X, Y) U, V, Z) \\
& -(\bar{\nabla} \alpha)(U, \tilde{I}(X, Y) V, Z)-(\bar{\nabla} \alpha)(U, V, \tilde{I}(X, Y) Z)
\end{aligned}
$$

respectively.

Theorem 4.1. On an invariant submanifold $\tilde{M}$ of a hyperbolic Sasakian manifold $M$, the condition $\bar{I}(X, Y) . \alpha=0$ holds if and only if it is totally geodesic provided that $\tilde{r} \neq n(n-1)$.

Proof: Suppose $\tilde{M}$ satisfies the condition $\bar{I}(X, Y) \cdot \alpha(U, V)=0$. Then from the equation (4.4), we have

$$
\tilde{R}^{\perp}(X, Y) \alpha(U, V)-\alpha(\tilde{I}(X, Y) U, V)-\alpha(U, \tilde{I}(X, Y) V)=0 .
$$

Putting $X=V=\xi$ in the above equation, we get

$$
\tilde{R}^{\perp}(\xi, Y) \alpha(U, \xi)-\alpha(\tilde{I}(\xi, Y) U, \xi)-\alpha(U, \tilde{I}(\xi, Y) \xi)=0,
$$

which, on using the equation (3.1), gives

$$
\alpha(U, \tilde{I}(\xi, Y) \xi)=0 .
$$

Using the equation (4.3) in the above equation, we get

$$
\left[1-\frac{\tilde{r}}{n(n-1)}\right] \alpha(Y, U)=0,
$$

which shows that $\alpha(U, Y)=0$, provided that $\tilde{r} \neq n(n-1)$. The converse part is trivial. This completes the proof.

Theorem 4.2. On an invariant submanifold $\tilde{M}$ of a hyperbolic Sasakian manifold $M$, the condition $\bar{I}(X, Y) \cdot \bar{\nabla} \alpha=0$ holds if and only if it is totally geodesic provided that $\tilde{r} \neq n(n-1)$. 
Proof: Suppose $\tilde{M}$ satisfies the condition $\bar{I}(X, Y) \cdot(\bar{\nabla} \alpha)(U, V, Z)=0$. Then in view of the equation (4.5), we have

$$
\begin{aligned}
& \tilde{R}^{\perp}(X, Y)(\bar{\nabla} \alpha)(U, V, Z)-(\bar{\nabla} \alpha)(\tilde{I}(X, Y) U, V, Z) \\
& -(\bar{\nabla} \alpha)(U, \tilde{I}(X, Y) V, Z)-(\bar{\nabla} \alpha)(U, V, \tilde{I}(X, Y) Z)=0 .
\end{aligned}
$$

Taking $X=V=\xi$ in the above equation, we get

$$
\begin{aligned}
& \tilde{R}^{\perp}(\xi, Y)(\bar{\nabla} \alpha)(U, \xi, Z)-(\bar{\nabla} \alpha)(\tilde{I}(\xi, Y) U, \xi, Z) \\
& -(\bar{\nabla} \alpha)(U, \tilde{I}(\xi, Y) \xi, Z)-(\bar{\nabla} \alpha)(U, \xi, \tilde{I}(\xi, Y) Z)=0 .
\end{aligned}
$$

Now, by virtue of the equations (2.28), (3.1), (4.2) and (4.3), we have the following equalities

$$
\begin{aligned}
(\bar{\nabla} \alpha)(\tilde{I}(\xi, Y) U, \xi, Z) & =\left(\bar{\nabla}_{\tilde{I}(\xi, Y) U} \alpha\right)(\xi, Z) \\
& =\tilde{\nabla}_{\tilde{I}(\xi, Y) U}^{\perp}(\alpha(\xi, Z))-\alpha\left(\tilde{\nabla}_{\tilde{I}(\xi, Y) U} \xi, Z\right)-\alpha\left(\xi, \tilde{\nabla}_{\tilde{I}(\xi, Y) U} Z\right) \\
& =-\alpha\left(\tilde{\nabla}_{\tilde{I}(\xi, Y) U} \xi, Z\right) \\
& =\alpha(\phi(\tilde{I}(\xi, Y) U), Z) \\
& =-\left[1-\frac{\tilde{r}}{n(n-1)}\right] \eta(U) \alpha(\phi Y, Z),
\end{aligned}
$$

$$
\begin{aligned}
(\bar{\nabla} \alpha)(U, \tilde{I}(\xi, Y) \xi, Z) & =\left(\bar{\nabla}_{U} \alpha\right)(\tilde{I}(\xi, Y) \xi, Z) \\
& =\tilde{\nabla}_{U}^{\perp}(\alpha(\tilde{I}(\xi, Y) \xi, Z))-\alpha\left(\tilde{\nabla}_{U}(\tilde{I}(\xi, Y) \xi), Z\right) \\
& -\alpha\left(\tilde{I}(\xi, Y) \xi, \tilde{\nabla}_{U} Z\right) \\
& =\tilde{\nabla}_{U}^{\perp}\left(\left(1-\frac{\tilde{r}}{n(n-1)}\right) \alpha(Y, Z)\right) \\
& -\alpha\left(\tilde{\nabla}_{U}\left(\left(1-\frac{\tilde{r}}{n(n-1)}\right)(\eta(Y) \xi+Y), Z\right)\right. \\
& -\left(1-\frac{\tilde{r}}{n(n-1)}\right) \alpha\left(Y, \tilde{\nabla}_{U} Z\right)
\end{aligned}
$$

and

$$
\begin{aligned}
(\bar{\nabla} \alpha)(U, \xi, \tilde{I}(\xi, Y) Z) & =\left(\bar{\nabla}_{U} \alpha\right)(\xi, \tilde{I}(\xi, Y) Z) \\
& =\tilde{\nabla}_{U}^{\perp}(\alpha(\xi, \tilde{I}(\xi, Y) Z))-\alpha\left(\tilde{\nabla}_{U} \xi, \tilde{I}(\xi, Y) Z\right) \\
& -\alpha\left(\xi, \tilde{\nabla}_{U} \tilde{I}(\xi, Y) Z\right) \\
& =-\left[1-\frac{\tilde{r}}{n(n-1)}\right] \eta(Z) \alpha(\phi U, Y) .
\end{aligned}
$$


Now substituting the equations (3.16), (4.12), (4.13) and (4.14) in the equation (4.11), we get

$$
\begin{aligned}
& \tilde{R}^{\perp}(\xi, Y) \alpha(\phi U, Z)+\left[1-\frac{\tilde{r}}{n(n-1)}\right] \eta(U) \alpha(\phi Y, Z)-\tilde{\nabla}_{U}^{\perp}\left(\left(1-\frac{\tilde{r}}{n(n-1)}\right) \alpha(Y, Z)\right) \\
& +\alpha\left(\tilde{\nabla}_{U}\left(\left(1-\frac{\tilde{r}}{n(n-1)}\right)(\eta(Y) \xi+Y), Z\right)+\left(1-\frac{\tilde{r}}{n(n-1)}\right) \alpha\left(Y, \nabla_{U} Z\right)\right. \\
& +\left[1-\frac{\tilde{r}}{n(n-1)}\right] \eta(Z) \alpha(\phi U, Y)=0 .
\end{aligned}
$$

Now taking $Z=\xi$ in the above equation and using the equations (3.1) and (3.3), we get

$$
\left[1-\frac{\tilde{r}}{n(n-1)}\right] \alpha(Y, \phi U)=0,
$$

which, by assuming $\tilde{r} \neq n(n-1)$, yields

$$
\alpha(Y, \phi U)=0 .
$$

Analogous to the proof of the previous theorem, we have $\alpha(Y, U)=0$, which shows that $\mathrm{M}$ is totally geodesic. The converse part is trivial. This completes the proof.

\section{Invariant Submanifold of Hyperbolic Sasakian Manifolds Satisfying $\bar{C}(X, Y) \cdot \alpha=0$ and $\bar{C}(X, Y) \cdot \bar{\nabla} \alpha=0$.}

The conformal curvature tensor $C$ of an n-dimensional Riemannian manifold is given by $[26]$

$$
\begin{gathered}
C(X, Y) Z=R(X, Y) Z-\frac{1}{(n-2)}[S(Y, Z) X-S(X, Z) Y+g(Y, Z) Q X-g(X, Z) Q Y] \\
+\frac{r}{(n-1)(n-2)}[g(Y, Z) X-g(X, Z) Y],
\end{gathered}
$$

for all vector fields $X, Y$ and $Z$ on $M$, where $S$ and $r$ are the Ricci tensor of type $(0,2)$ and the scalar curvature respectively of $M$.

Putting $X=\xi$ in the above equation and using the equations (2.7), (3.5) and (3.7), we get

(5.2) $\tilde{C}(\xi, Y) Z=\frac{\tilde{r}-(n-1)}{(n-1)(n-2)}[g(Y, Z) \xi-\eta(Z) Y]-\frac{1}{(n-2)}[\tilde{S}(Y, Z) \xi-\eta(Z) \tilde{Q} Y]$,

which, on putting $Z=\xi$ and by use of the equation (2.4), gives

$$
\tilde{C}(\xi, Y) \xi=\frac{(n-1)(n-2)+\tilde{r}}{(n-1)(n-2)}[\eta(Y) \xi+Y]
$$


where $\tilde{C}$ is the conformal curvature tensor of $\tilde{M}$.

In view of the equations (2.32) and (2.33), the tensors $\bar{C}(X, Y) \cdot \alpha$ and $\bar{C}(X, Y) \cdot \bar{\nabla} \alpha$ are defined by [18]

$$
(\bar{C}(X, Y) \cdot \alpha)(U, V)=\tilde{R}^{\perp}(X, Y) \alpha(U, V)-\alpha(\tilde{C}(X, Y) U, V)-\alpha(U, \tilde{C}(X, Y) V)
$$

and

$$
\begin{aligned}
(\bar{C}(X, Y) \cdot \bar{\nabla} \alpha)(U, V, Z) & =\tilde{R}^{\perp}(X, Y)(\bar{\nabla} \alpha)(U, V, Z)-(\bar{\nabla} \alpha)(\tilde{C}(X, Y) U, V, Z) \\
& -(\bar{\nabla} \alpha)(U, \tilde{C}(X, Y) V, Z)-(\bar{\nabla} \alpha)(U, V, \tilde{C}(X, Y) Z)
\end{aligned}
$$

respectively.

Theorem 5.1. On an invariant submanifold $\tilde{M}$ of a hyperbolic Sasakian manifold $M$, the condition $\bar{C}(X, Y) . \alpha=0$ holds if and only if it is totally geodesic provided that $\tilde{r} \neq-(n-1)(n-2)$.

Proof: Suppose $\tilde{M}$ satisfies the condition $(\bar{C}(X, Y) \cdot \alpha)(U, V)=0$. Then from the equation (5.4), we have

$$
\tilde{R}^{\perp}(X, Y) \alpha(U, V)-\alpha(\tilde{C}(X, Y) U, V)-\alpha(U, \tilde{C}(X, Y) V)=0 .
$$

Putting $X=V=\xi$ in the above equation, we get

$$
\tilde{R}^{\perp}(\xi, Y) \alpha(U, \xi)-\alpha(\tilde{C}(\xi, Y) U, \xi)-\alpha(U, \tilde{C}(\xi, Y) \xi)=0,
$$

which, on using the equation (3.1), gives

$$
\alpha(U, \tilde{C}(\xi, Y) \xi)=0 .
$$

Using the equation (5.3) in the above equation, we get

$$
\left[\frac{\tilde{r}+(n-1)(n-2)}{(n-1)(n-2)}\right] \alpha(Y, U)=0,
$$

which shows that $\alpha(U, Y)=0$, provided that $\tilde{r} \neq-(n-1)(n-2)$. The converse statement is trivial. This completes the proof.

Theorem 5.2. On an invariant submanifold $\tilde{M}$ of a hyperbolic Sasakian manifold $M$, the condition $\bar{C}(X, Y) \cdot \bar{\nabla} \alpha=0$ holds if and only if it is totally geodesic provided that $\tilde{r} \neq(n-1)$.

Proof: Suppose $\tilde{M}$ satisfies the condition $\bar{C}(X, Y) \cdot(\bar{\nabla} \alpha)(U, V, Z)=0$. Then in view of the equation (5.5), we have

$$
\begin{aligned}
& \tilde{R}^{\perp}(X, Y)(\bar{\nabla} \alpha)(U, V, Z)-(\bar{\nabla} \alpha)(\tilde{C}(X, Y) U, V, Z) \\
& -(\bar{\nabla} \alpha)(U, \tilde{C}(X, Y) V, Z)-(\bar{\nabla} \alpha)(U, V, \tilde{C}(X, Y) Z)=0 .
\end{aligned}
$$


Putting $X=V=\xi$ in the above equation, we get

$$
\begin{aligned}
& \tilde{R}^{\perp}(\xi, Y)(\bar{\nabla} \alpha)(U, \xi, Z)-(\bar{\nabla} \alpha)(\tilde{C}(\xi, Y) U, \xi, Z) \\
& -(\bar{\nabla} \alpha)(U, \tilde{C}(\xi, Y) \xi, Z)-(\bar{\nabla} \alpha)(U, \xi, \tilde{C}(\xi, Y) Z)=0 .
\end{aligned}
$$

Now, by virtue of the equations $(2.28),(3.1),(5.2)$ and (5.3), we have the following equations

$$
\begin{aligned}
& (\bar{\nabla} \alpha)(\tilde{C}(\xi, Y) U, \xi, Z)=\left(\bar{\nabla}_{\tilde{C}(\xi, Y) U} \alpha\right)(\xi, Z) \\
& =\tilde{\nabla} \tilde{\tilde{C}}_{(\xi, Y) U}^{\perp}(\alpha(\xi, Z))-\alpha\left(\tilde{\nabla}_{\tilde{C}(\xi, Y) U} \xi, Z\right) \\
& -\alpha\left(\xi, \tilde{\nabla}_{\tilde{C}(\xi, Y) U} Z\right) \\
& =\alpha(\phi(\tilde{C}(\xi, Y) U), Z) \\
& =\left[\frac{n(n-1)-\tilde{r}}{(n-1)(n-2)}\right] \eta(U) \alpha(\phi Y, Z), \\
& (\bar{\nabla} \alpha)(U, \tilde{C}(\xi, Y) \xi, Z)=\left(\bar{\nabla}_{U} \alpha\right)(\tilde{C}(\xi, Y) \xi, Z) \\
& =\tilde{\nabla}_{U}^{\perp}(\alpha(\tilde{C}(\xi, Y) \xi, Z))-\alpha\left(\tilde{\nabla}_{U}(\tilde{C}(\xi, Y) \xi), Z\right) \\
& -\alpha\left(\tilde{C}(\xi, Y) \xi, \tilde{\nabla}_{U} Z\right) \\
& =\tilde{\nabla}_{U}^{\perp}\left(\left(\frac{\tilde{r}+(n-1)(n-2)}{(n-1)(n-2)}\right) \alpha(Y, Z)\right) \\
& -\alpha\left(\tilde{\nabla}_{U}\left(\left(\frac{\tilde{r}+(n-1)(n-2)}{(n-1)(n-2)}\right)(\eta(Y) \xi+Y), Z\right)\right. \\
& -\left(\frac{\tilde{r}+(n-1)(n-2)}{(n-1)(n-2)}\right) \alpha\left(Y, \tilde{\nabla}_{U} Z\right)
\end{aligned}
$$

and

$$
\begin{aligned}
(\bar{\nabla} \alpha)(U, \xi, \tilde{C}(\xi, Y) Z) & =\left(\bar{\nabla}_{U} \alpha\right)(\xi, \tilde{C}(\xi, Y) Z) \\
& =\tilde{\nabla}_{U}^{\perp}(\alpha(\xi, \tilde{C}(\xi, Y) Z))-\alpha\left(\tilde{\nabla}_{U} \xi, \tilde{C}(\xi, Y) Z\right)-\alpha\left(\xi, \tilde{\nabla} \tilde{C}_{\tilde{C}}(\xi, Y) Z\right) \\
& \left.=\frac{n(n-1)-\tilde{r}}{(n-1)(n-2)}\right] \eta(Z) \alpha(\phi U, Y) .
\end{aligned}
$$

In view of the equations $(3.16),(5.12),(5.13)$ and (5.14), the equation (5.11) takes the form

$$
\begin{aligned}
& \tilde{R}^{\perp}(\xi, Y) \alpha(\phi U, Z)+\left[\frac{n(n-1)-\tilde{r}}{(n-1)(n-2)}\right] \eta(U) \alpha(\phi Y, Z) \\
& -\tilde{\nabla}_{U}^{\perp}\left(\left(\frac{\tilde{r}+(n-1)(n-2)}{(n-1)(n-2)}\right) \alpha(Y, Z)\right)+\alpha\left(\tilde{\nabla}_{U}\left(\left(\frac{\tilde{r}+(n-1)(n-2)}{(n-1)(n-2)}\right)(\eta(Y) \xi+Y), Z\right)\right. \\
& \left.+\left(\frac{\tilde{r}+(n-1)(n-2)}{(n-1)(n-2)}\right) \alpha\left(Y, \tilde{\nabla}_{U} Z\right)-\frac{n(n-1)-\tilde{r}}{(n-1)(n-2)}\right] \eta(Z) \alpha(\phi U, Y)=0,
\end{aligned}
$$


which, on taking $Z=\xi$ and by use of the equations (3.1) and (3.3), gives

$$
\frac{(n-1)-\tilde{r}}{(n-1)(n-2)} \alpha(Y, \phi U)=0 .
$$

Now putting $U=\phi U$ in the above equation and using the equations (2.1) and (3.1), we get

$$
\frac{(n-1)-\tilde{r}}{(n-1)(n-2)} \alpha(Y, U)=0,
$$

which yields by assuming $\tilde{r} \neq(n-1)$,

$$
\alpha(Y, U)=0 .
$$

This shows that $\tilde{M}$ is totally geodesic. The converse part is trivial. This completes the proof.

\section{R E F E R E N C E S}

1. K. Arslan, $\ddot{U}$. Lumiste, C. Murathan and C. ÖzGür: 2-semiparallel surfaces in space forms I. Two perticular cases, Proc. Estonian Acad. Sci. Phys. Math., 49(3) (2000), 139-148.

2. A. BejAnCu and N. PAPAghuic: Semi-invariant submanifolds of a Sasakian manifold, An Stiint. Univ. "AL I CUZA" Iasi, 27 (1981), 163-170.

3. D. E. Blair and G. D. Ludden: Hypersurfaces in almost contact manifolds, Tohoku Math. J., 22 (1969), 354-362.

4. D. E. Blair, Contact manifolds in Riemannian Geometry, Lecture notes in Math., 509, Springer Verlag, Verlin, 1976.

5. B. Y. Chen: Goemetry of submanifolds, Marcel Dekker, New York, 1973.

6. U. C. DE and A. A. SHAIKH: Non-existance of proper semi-invariant submanifold of a Lorentzian para-Sasakian manifold, Bull. Malayasian Math.Soc.(Second series), 22(1999), 179-183.

7. U. C. De and A. K. Sengupta: CR-Submanifolds of a Lorentzian para-Sasakian manifold, Bull. Malayasian Math.Soc., 23 (2000), 99-106.

8. U. C. De, A. Al- Aqueal and A. A. Shaikh: Submanifolds of Lorentzian paraSasakian manifold, Bull. Malayasian Math.Soc., 28 (2005), 223-227.

9. U. C. DE and A. SARKAR: On pseudo-slant submanifolds of trans-Sasakian manifolds, Proc. Estonian Acad. Sci., 60 (2011), 1-11.

10. J. Deprez: Semi-parallel surfaces in the Euclidean space, Journal of Geometry, 25 (1985), 192-200.

11. S. I. Goldberg and K. YAno: Non-invariant hypersurfaces of almost contact manifolds, J. Math. Soc. of Japan, 22(1) (1970), 25-34.

12. S. I. GoldBerg: Invariant submanifolds of co-dimension 2 of almost contact manifolds, Scuola Nomale Superiore, 25Fasc (3)(1971), 377-388.

13. Z. GuOJIng and W. JiAngu: Invariant submanifolds and modes of non-linear autonomous system, Applied Mathematics and Mechanics, 19 (1998), 687-693. 
14. K. Matsumoto, I. Minai and R. RosacA: $\xi$-null geodesic vector fields on a LP-Sasakian manifold, J.Korean Math.Soc.,32 (1995), 17-31.

15. R. S. Mishra: Submanifolds of a locally product Riemannian manifold, Canad. Math. Bull., 11 (1968), 423-435.

16. R. S. Mishra: Almost complex and almost contact submanifols, Tensor N.S., 25 (1972), 419-433.

17. R. S. Mishra and M. P. RAthore: On framed mertic submanifolds, The Yokohama Math. J., 24(1\& 2) (1976), 13-20.

18. C. ÖzGür and C. Murathan: On invariant submanifolds of Lorentzian paraSasakian manifolds, The Arabian Journal for Science and Engineering, 34(2A) (2008), 177-185.

19. N. PAPAGhuiC: Semi-invariant submanifolds in a Kenmotsu manifold, Rend. Mat., 3 (1983), 607-622.

20. A. SARKAR: On submanifolds of Sasakian manifolds, Lobachevskii J.Math., 32 (2011), 87-93.

21. A. Sarkar and M. Sen: On invariant submanifolds of trans-Sasakian manifolds, Proc. Estonian Acad. Sci., 61(1) (2012), 29-37.

22. R. N. Singh, S. K. PANDey and G. PANDEy: On submanifolds of hyperbolic Sasakian manifolds, (Communicated).

23. B. B. Sinha and R. SHARMA: Hypersurfaces in an almost paracontact manifold, Indian J.Pure Appl.Math., 9 (1978), 1083-1090.

24. S. Sular and C. Özgur: On submanifolds of Kenmotsu manifolds, Chaos, Solitons and Fractals, 42 (2009), 1990-1995.

25. M. D. Upadhyay and K. K. Dubey: Almost hyperbolic contact $(f, \xi, \eta, g)$ structure, Acta Mathematica Academaiae Scientiarum Hungrical Tomus, 28 H-1053, 13-15.

26. K. YAno and M. Kon: Structures on manifolds, Series in Pure Mathematics (World Scientific, Singapore), 1984.

Shravan Kumar Pandey

Department of Mathematical Sciences

A.P.S.University, Rewa (M.P.) India

shravan.math@gmail.com

Ram Nawal Singh

Department of Mathematical Sciences

A.P.S.University, Rewa (M.P.) India

rnsinghmp@rediffmail.com 